\title{
Avaliação Genética de Touros pelo Modelo Animal, Modelo Touro e Método das Companheiras de Rebanho ${ }^{1}$
}

\author{
Rui da Silva Verneque ${ }^{2,3}$, Mário Luiz Martinez 2,3 , Roberto Luiz Teodoro ${ }^{2,3}$, Amaury Alípio \\ Pimentel $^{3}$, William José Ferreira ${ }^{3}$
}

\begin{abstract}
RESUMO - As capacidades previstas de transmissão (PTA) para a produção de leite de 529 touros, usando o modelo animal (MA), modelo touro (MT) e o método das companheiras de rebanho (MCR), foram calculadas e comparadas por meio da correlação de ordem e valores, da confiabilidade e das diferenças entre PTA e percentual de coincidência na seleção dos melhores touros. Considerando-se dois conjuntos de dados, um contendo até cinco lactações por vaca e outro com os dados de produção à primeira lactação, analisaramse 12.762 lactações, de 5757 vacas da raça Gir e mestiças Gir-Holandês. Admitindo-se até cinco lactações por vaca, a correlação de ordem entre PTA previstas usando-se MA e MCR foi de 0,69; entre modelo animal e modelo touro, 0,96; e entre MCR e modelo touro, 0,73. As correlações de ordem entre as PTA usando-se MA, com até cinco lactações por vaca, e as PTA previstas pelo MCR, MT e MA, utilizando-se apenas a primeira lactação, foram 0,60;0,76; e 0,79, respectivamente. Maiores médias de confiabilidade das PTA foram obtidas quando se usaram modelo animal e modelo touro, os quais foram semelhantes na seleção dos melhores touros, segundo os valores de PTA. A confiabilidade da prova estimada quando se usou modelo animal foi 11 a 47\% maior que a estimada pelo MCR. Estas diferenças foram de, no máximo, $2 \%$, quando se compararam os modelos animal e touro.
\end{abstract}

Palavras-chave: avaliação genética, gado de leite, método das companheiras de rebanho, modelo animal, modelos mistos, modelo touro, raça Gir

\section{Sire Evaluation by Animal Model, Sire Model and Contemporary Herdmate Method}

ABSTRACT - Predicted transmitting ability (PTA) of milk yield of 529 sires using animal model (MA), sire model (MT) and herdmate contemporary comparison method (MCR) were calculated and compared by means of correlation's rank and values, reliability, differences among PTA and percent of coincidence in the selection of the best bulls. Two sets of data were considered one contained up to five lactation per cow and other with production data at first lactation. Data of 12,762 lactations of 5757 Gyr and crossbred Gyr Holstein cows were analyzed. Allowing up to five lactation per cow, the rank correlation among PTA calculated using MA and MCR was .69; between MA and MT was .96; and between MT and MCR was .73. The rank correlation between PTA using MA up to five lactation per cow, and PTA calculated by MCR, MT and MA using only the first lactation, were: .60, .76, and .79, respectively. Average reliabilities were greater when animal and sire model were used. These two models were similar in the selection of the best sires according to the PTA values. The estimate reliability when the animal model were used was 11 to $47 \%$ greater than the estimated by MCR. These maximum differences were $2 \%$ when animal model was compared with sire model.

Key Words: genetic evaluation, dairy cattle, contemporary herdmates, animal model, mixed model, sire model, Gyr breed

\section{Introdução}

A avaliação genética é um processo que consiste em prever o valor genético dos animais, compatível com as restrições impostas pela estrutura dos dados disponíveis, pela metodologia adotada e pelos recursos computacionais existentes (VERNEQUE, 1994). Em gado de leite, a avaliação genética é importante na identificação de animais de genética aditiva superior. Assim, o uso de metodologias adequadas nas avaliações genéticas é fundamental para o melhoramento, pois os seus resultados possibilitam identificar com maior precisão os melhores animais que servirão como pais das futuras gerações.

No método das companheiras de rebanho (MCR) (DOMMERHOLT, 1982) o touro é avaliado com base no desvio das produções de leite de suas filhas em relação às companheiras de rebanho. Para isso, é necessário ajuste prévio dos dados para as causas de variação não-genéticas, denominadas efeitos fixos, representados principalmente por rebanho-ano-época, composição genética e idade da vaca ao parto. Por outro lado, usando-se modelos lineares mistos (HENDERSON, 1973), para avaliação de touros, é

\footnotetext{
1 Trabalho executado entre EMBRAPA Gado de Leite e ABCGIL.

2 Pesquisador da EMBRAPA Gado de Leite - Rua Eugênio do Nascimento, 610 - 36038-330 - Juiz de Fora - MG.

${ }^{3}$ Bolsista do CNPq.
} 
possível estabelecer vários tipos de modelos, dos quais dois são os mais importantes. $\mathrm{O}$ primeiro considera touro como efeito aleatório principal, cujo componente de variância representa $1 / 4$ do efeito genético aditivo para a característica e no segundo o efeito é o próprio animal (vaca, touro ou mãe), em que o componente de variância é o efeito genético aditivo direto. Os dois tipos de modelos são comumente denominados, respectivamente, de modelo touro (MT) e modelo animal (MA) (HENDERSON, 1988). Desde 1989, o MA tornou-se a base para a avaliação genética nos Estados Unidos (VAN VLECK, 1992). Este modelo permite avaliar touros e vacas simultaneamente. Tanto o MA quanto o MT agregam informações de pedigree para avaliação do animal, contribuindo para o aumento da confiabilidade do valor genético previsto. Além disso, os dados são simultaneamente ajustados para todos os efeitos incluídos no modelo, sendo desnecessário o ajuste prévio dos dados para os efeitos fixos.

Em geral, quando há muitas informações disponíveis no pedigree, ou seja, muitos parentes compõem a matriz de parentesco, espera-se que a avaliação pelo modelo animal apresente maior confiabilidade que a avaliação pelo método das companheiras de rebanho ou modelo touro. Por outro lado, quando há muita informação de pedigree para os animais avaliados ou o número de filhas por touro é grande, pelo MT pode-se obter previsores com a mesma confiabilidade do MA. SCHAEFFER (1996) cita que para herdabilidades variando de 0,10 a 0,50 , vinte filhas por touro têm peso de 77 a $82 \%$ sobre a avaliação do reprodutor. Dessa forma, informações de muitas filhas, distribuídas em diversos rebanhos, reduzem o peso das informações adicionais de pedigree para avaliação do touro.

Algumas das limitações ao uso do MA são o alto requerimento de recursos computacionais, especialmente memória RAM e o tempo de processamento gasto para a avaliação genética, que, em geral, é elevado, especialmente quando se trabalha com muitos animais, e o grande número de classes de efeitos fixos ou mais de uma característica avaliada ao mesmo tempo. Por outro lado, o MT requer menor tempo de processamento e as exigências de memória RAM são muito menores. Assim, para grandes massas de dados, com pedigree incompleto, essa pode ser alternativa viável, especialmente quando se dispõe de recursos computacionais limitados.

Apesar de existirem poucos sistemas disponíveis e suficientemente testados que adotam MA e MT para avaliação genética de animais, considerando-se informações de pedigree, a tendência é a utilização de um desses dois modelos em todo mundo.

NORMAN et al. (1991), comparando o MCR com o MA, observaram que a confiabilidade dos valores genéticos previstos pelo MA foi de 13 a 17\% maior que a estimada pelo MCR. A capacidade de prever o mérito genético de futuras filhas e filhos foi de 3 a $5 \%$ superior no MA em relação ao MCR. Os valores da PTA foram de 4 a $11 \%$ maiores quando se usou MA em vez do MCR. Da mesma forma, VAN RADEN et al. (1989) concluíram que a previsão da performance da progênie é mais confiável utilizando-se o MA que o MCR. NORMAN et al. (1991), em estudo comparativo entre os dois procedimentos, encontraram correlação de ordem entre os valores genéticos previstos pelo MA e MCR de 0,92 a 0,95 e diferenças absolutas entre os valores genéticos previstos pelo MA e MCR decresceram para confiabilidades acima de $90 \%$. Além disso, esses autores verificaram que a confiabilidade da prova foi cerca de $4 \%$ maior, na avaliação de vacas e touros, quando se utilizaram mais informações de pedigree, adotando-se modelo animal. VERNEQUE et al. (1996) encontraram correlação de ordem de 0,84 entre os valores genéticos de touros, previstos pelo MA e MCR, e RUVUNA et al. (1979) estimaram a correlação de ordem entre os valores genéticos previstos pelo MCR e MT em 0,79.

O objetivo deste estudo foi comparar os valores genéticos para a produção de leite obtidos pelo modelo animal, modelo touro e método das companheiras de rebanho, por intermédio dos dados de produção de leite de rebanhos Gir leiteiro e seus mestiços.

\section{Material e Métodos}

As informações utilizadas neste estudo foram obtidas do programa de teste de progênie de touros da raça Gir. Foram usados os dados acumulados durante 31 anos, com partos registrados entre 1965 e 1996, totalizando 19.283 lactações, de 9046 vacas, filhas de 795 touros. Previamente às avaliações genéticas, realizou-se a consistência dos dados, excluindo as lactações com causa de encerramento anormal, como: morte do bezerro ou morte, venda ou doença da vaca antes do encerramento da lactação. Também foram consideradas anormais, e portanto excluídas, as lactações com duração inferior a 90 ou superior a 600 dias. Além disso, para a avaliação usando-se o MA e MT utilizaram-se apenas os registros de produção de vacas que tiveram a primeira lactação controlada e classe de rebanho-ano de parto que continham no mínimo três animais. Ao final do processo de consis- 
306

Rev. bras. zootec.

tência dos dados, resultaram 12.762 lactações, de 5757 vacas, filhas de 529 reprodutores.

$\mathrm{Na}$ avaliação genética de touros adotando-se o MCR, utilizou-se o programa desenvolvido por DOMMERHOLT (1982). Foram consideradas até cinco lactações de cada vaca. Os dados de produção de leite em até 305 dias de lactação foram previamente ajustados para idade adulta e para os efeitos de época de parto e composição genética da vaca. Utilizaram-se herdabilidade e repetibilidade para produção de leite de 0,25 e 0,45 , respectivamente. Neste método, os valores genéticos (VG) dos touros são previstos por meio da expressão:

$$
V G=\mathrm{b} \frac{1}{\mathrm{n}} \sum_{i=1}^{n} D V_{\mathrm{i}}
$$

em que

$$
\mathrm{b}=\frac{2 \sum_{\mathrm{i}} w_{i} h^{2}}{4+\sum\left(\mathrm{w}_{\mathrm{i}}-1\right) \mathrm{h}^{2}+\frac{4 \sum \mathrm{n}_{\mathrm{i}}\left(n_{i}-1\right) \mathrm{c}^{2}}{N}},
$$

$\mathrm{DV}_{\mathrm{i}}$ é o desvio da média de produção de leite da filhai do touro em relação às companheiras de rebanho, acrescida de $10 \%$ das diferenças entre rebanhos que são presumidas genéticas (DOMMERHOLT, 1982);

b é o coeficiente de regressão do valor genético verdadeiro sobre a média dos desvios das filhas;

n é o número de lactações das filhas de um reprodutor;

$$
\mathrm{W}_{\mathrm{i}}=\frac{\mathrm{m}_{\mathrm{i}}}{1+\left(\mathrm{m}_{\mathrm{i}}-1\right) \mathrm{r}} \begin{aligned}
& \begin{array}{l}
\text { é fator de ponderação para } \\
\text { cada vaca, de acordo com o } \\
\text { número de lactações; }
\end{array}
\end{aligned}
$$

em que

$\mathrm{m}_{\mathrm{i}}$ é o número de lactações da i-ésima vaca;

$r$ é a repetibilidade da produção de leite $(0,45)$;

$\mathrm{N}$ é o número total de progênies (filhas dos reprodutores);

$\mathrm{n}_{\mathrm{i}}$ é o número de progênies do reprodutor no iésimo rebanho;

$\mathrm{h}^{2}$ é a heritabilidade da produção de leite $(0,25)$; e

$\mathrm{c}^{2}$ é a correlação residual entre meio-irmãs paternas em um mesmo rebanho $(0,15)$.

A capacidade prevista de transmissão (PTA) da produção de leite é a metade do valor genético (VG).

A confiabilidade da prova é calculada como:

$$
R E L=\sqrt{\frac{1}{2} b}
$$

Nas avaliações usando-se MA ou MT, o sistema utilizado foi o MTDFREML (BOLDMAN et al.,
1995). O modelo incluiu como efeitos fixos rebanhoano, época de parto (1 - águas de outubro a março e 2 - seca de abril a setembro), composição genética (Gir, 1/8, 1/4, 3/8 e 1/2 Holandês-Gir) e co-variável idade da vaca ao parto, com termos linear e quadrático. Além do erro, foi incluído no MA o animal como efeito aleatório, representando efeito genético aditivo que incluiu 9.095 indivíduos (vaca, pai e mãe), e para MT, o efeito de touro, que incluiu 761 animais (529 touros e mais a extensão de pedigree). Para os dois modelos foi também incluído o efeito permanente de meio representado pelas medidas repetidas de uma mesma vaca que produziu leite.

Os modelos estatísticos usados tanto para modelo animal quanto para modelo touro na forma matricial foram:

$$
\mathrm{y}=\mathrm{X} \beta+\mathrm{Z} 1 \mathrm{u}+\varepsilon
$$

quando se trabalhou com a produção à primeira lactação ou

$$
\mathrm{y}=\mathrm{X} \beta+\mathrm{Z} 1 \mathrm{u}+\mathrm{Z} 2 \mathrm{p}+{ }_{\varepsilon}
$$

quando considerou mais de uma lactação por vaca. em que

y é um vetor contendo as produções de leite em até 305 dias de lactação de cada vaca;

$\mathrm{X}$ é a matriz de incidência dos efeitos fixos (rebanho-ano, época, composição genética e idade da vaca ao parto);

$ß$ é um vetor de parâmetros desconhecidos representando os efeitos fixos;

$Z_{1}$ e $Z_{2}$ são matrizes de incidência dos efeitos aleatórios de animal e efeito permanente de meio, respectivamente;

u é um vetor de classes de efeitos aleatórios, que incluiu os valores genéticos previstos dos animais que estavam sendo avaliados (touros ou animais);

p é um vetor de efeito permanente de meio, decorrente de registros repetidos de uma mesma vaca ou da produção de leite de filhas de um mesmo touro; e

$\varepsilon$ é um vetor dos erros aleatórios.

Os valores genéticos previstos por animal foram estimados por meio da maximização do logarítimo da função de verossimilhança conjunta dos vetores $\mathrm{y}, \mathrm{u}$ e $p$ (BOLDMAN e VAN VLECK, 1991 e MEYER,1988), obtendo-se as equações para û.

A capacidade prevista de transmissão (PTA) para cada animal corresponde à metade de û.

A confiabilidade (REL) para cada valor genético foi estimada por:

$$
R E L=\sqrt{1-\frac{P E V}{\hat{\sigma}_{a}^{2}(1+F A)}}
$$


em que

$\hat{\sigma}_{a}^{2}$ é a estimativa da variância dos efeitos genéticos aditivos de animal;

FA é o coeficiente de consangüinidade do animal, cujo valor genético está sendo predito;

PEV é a variância do erro de predição do valor genético do animal, calculada como: $\mathrm{PEV}=\mathrm{C}-{ }^{1} \mathrm{k}$, com C- ${ }^{1}$ a inversa da matriz de coeficientes das equações do modelo misto (MME); e

$\mathrm{k}$ é um vetor de mesma ordem da matriz $\mathrm{C}$, com 1 na linha correspondente ao animal e 0 nas demais linhas. Na realidade, para estimar PEV não se inverte a matriz $\mathrm{C}$, mas a solução é obtida pelo mesmo processo de estimação do valor genético dos animais usando a decomposição de Cholesky da matriz C (VERNEQUE, 1994 e BOLDMAN et al., 1995).

As avaliações genéticas foram realizadas considerando-se a produção de leite de até cinco lactações por vaca. Neste caso, usou-se o modelo (2). Quando se usou o modelo animal ou modelo touro, toda vaca obrigatoriamente tinha que apresentar produção à primeira lactação. Também foram realizadas avaliações considerando-se apenas esta produção. Neste caso, foi utilizado o modelo (1).

A comparação das metodologias e dos procedimentos para avaliação genética de touros foi realizada por intermédio da análise de:

- Correlações de ordem (método de Spearman) e de valores (método de Pearson) (CONOVER, 1980) entre as PTA calculadas pelos diferentes procedimentos;

- Variação média nos valores das PTA por classe de confiabilidade;

- Médias de confiabilidade usando-se os diferentes procedimentos;

- Desvio absoluto entre as PTA calculadas usando-se MA e PTA calculadas por intermédio do modelo touro ou método das companheiras de rebanho;

- Número e porcentagem de touros alocados por classe de diferença absoluta entre valores das PTA; e

- Porcentagem de coincidência de touros que seriam selecionados pelos diferentes procedimentos, em relação ao percentual de touros selecionados usando-se modelo animal, considerando até cinco lactações por vaca.

\section{Resultados e Discussão}

Nas Tabelas 1 e 2 são apresentadas as médias e respectivos desvios-padrão das PTA e confiabilidades (REL) estimadas usando-se modelo animal (MA), considerando produções de leite em até cinco lactações por vaca (PTA5 $5_{\text {animal }}$ e REL5 $5_{\text {animal }}$ ), produção na primeira lactação (PTA $1_{\text {animal }}$ e REL1 ${ }_{\text {animal }}$ ), método das companheiras de rebanhos (MCR), com até cinco lactações por vaca (PTA5 ${ }_{\text {mcr }}$ e REL5 ${ }_{\text {mcr }}$ ) ou produção na primeira lactação $\left(\right.$ PTA $1_{\text {mcr }}$ e REL $1_{\text {mcr }}$ ) e modelo touro (MT) com até cinco lactações (PTA5 touro REL $_{\text {touro }}$ ) ou a produ-

Tabela 1 -Médias estimadas das PTA de touros, usando-se diferentes procedimentos de avaliação, por classe de confiabilidade estimada usando modelo animal 1

Table 1 - Estimated means for PTA of sires using different procedures of evaluation by classe of reliability estimated using animal model Classe de confiabilidade $\quad \mathrm{N}^{0}$ de touros

Class of reliability No. of sires Média \pm Desvio-padrão

\begin{tabular}{|c|c|c|c|c|c|c|c|}
\hline & & PTA5 animal & $\mathrm{PTA5}_{\mathrm{mcr}}$ & PTA5 ${ }_{\text {touro }}$ & PTA $1_{\text {animal }}$ & $\mathrm{PTA}_{\mathrm{mcr}}$ & $\overline{\text { PTA } 1_{\text {touro }}}$ \\
\hline$<.20$ & 4 & $-8,5 \pm 056$ & $-26,6 \pm 96$ & $-8,6 \pm 53$ & $-11,2 \pm 66$ & $-43,7 \pm 076$ & $-8,5 \pm 049$ \\
\hline $.20-.30$ & 152 & $-2,6 \pm 047$ & $-8,1 \pm 56$ & $-3,9 \pm 44$ & $-2,1 \pm 41$ & $-5,1 \pm 043$ & $-2,2 \pm 030$ \\
\hline $.30-.40$ & 78 & $-12,1 \pm 083$ & $-20,0 \pm 73$ & $-13,8 \pm 80$ & $-8,1 \pm 64$ & $-13,7 \pm 059$ & $-7,3 \pm 046$ \\
\hline $.40-.50$ & 58 & $-2,9 \pm 109$ & $-13,3 \pm 82$ & $-11,4 \pm 101$ & $-4,0 \pm 93$ & $-8,9 \pm 063$ & $-9,5 \pm 065$ \\
\hline $.50-.60$ & 56 & $20,0 \pm 119$ & $15,6 \pm 109$ & $14,3 \pm 114$ & $9,9 \pm 105$ & $-4,8 \pm 079$ & $4,2 \pm 078$ \\
\hline $.60-.70$ & 58 & $5,4 \pm 115$ & $-6,8 \pm 87$ & $1,1 \pm 133$ & $7,4 \pm 128$ & $-12,6 \pm 081$ & $2,2 \pm 095$ \\
\hline $.70-.80$ & 44 & $16,3 \pm 164$ & $-7,9 \pm 75$ & $5,8 \pm 152$ & $21,3 \pm 123$ & $-15,2 \pm 069$ & $10,7 \pm 100$ \\
\hline $.80-.90$ & 69 & $125,7 \pm 123$ & $2,6 \pm 99$ & $104,0 \pm 117$ & $82,5 \pm 112$ & $-9,2 \pm 092$ & $55,7 \pm 096$ \\
\hline$>.90$ & 10 & $137,9 \pm 140$ & $48,8 \pm 64$ & $152,9 \pm 124$ & $87,4 \pm 148$ & $26,6 \pm 062$ & $83,9 \pm 136$ \\
\hline Média & 529 & $19,7 \pm 115$ & $-5,6 \pm 80$ & $13,7 \pm 107$ & $13,7 \pm 96$ & $-8,5 \pm 066$ & $7,3 \pm 074$ \\
\hline
\end{tabular}

Mean

\footnotetext{
${ }_{1 \text { PTA5 }}^{\text {animal }}=$ Capacidade prevista de transmissão (PTA) usando modelo animal e até cinco lactações por vaca (PTA using animal model and up to five lactation per cow).

$\mathrm{PTA5}_{\mathrm{mcr}}=\mathrm{PTA}$ usando o método das companheiras de rebanho e até cinco lactações por vaca (PTA using a herdmate comparison method and up to five lactation per cow).

PTA5 $_{\text {touro }}=$ PTA usando modelo touro e até cinco lactações por vaca (PTA using sire model and up to five lactacions per cow).

PTA 1 animal = PTA usando modelo animal e a produção de leite na primeira lactação (PTA using animal model and only the first lactation).

PTA 1 mcr = PTA usando MCR e apenas a produção de leite na primeira lactação (PTA using the herdmate comparison method and only the first lactation).

PTA $1_{\text {touro }}=$ PTA usando modelo touro e a produção de leite na primeira lactação (PTA using sire model and the milk production in the first lactation).
} 
308 Rev. bras. zootec.

Tabela 2 - Médias da confiabilidade, estimada para os diferentes procedimentos, por classes de confiabilidade calculada usando modelo animal ${ }^{1}$

Table 2 - Means for reliability, estimated for the different procedures, by classes of reliability calculated using animal model Classe de confiabilidade Confiabilidade

Class of reliability Reliability

\begin{tabular}{lcccccr} 
& REL5 $_{\mathrm{mcr}}$ & REL1 $_{\mathrm{mcr}}$ & REL5 $_{\text {animal }}$ & REL1 $_{\text {animal }}$ & REL5 $_{\text {touro }}$ & REL1 $_{\text {touro }}$ \\
\hline$<.20$ & $0,08 \pm 0,03$ & $0,05 \pm 0,00$ & $0,19 \pm 0,00$ & $0,18 \pm 0,00$ & $0,19 \pm 0,00$ & $0,16 \pm 0,00$ \\
$.20-.30$ & $0,08 \pm 0,04$ & $0,05 \pm 0,01$ & $0,25 \pm 0,03$ & $0,23 \pm 0,03$ & $0,23 \pm 0,02$ & $0,19 \pm 0,02$ \\
$.30-.40$ & $0,12 \pm 0,05$ & $0,09 \pm 0,03$ & $0,34 \pm 0,03$ & $0,32 \pm, 003$ & $0,31 \pm 0,04$ & $0,26 \pm 0,05$ \\
$.40-.50$ & $0,17 \pm 0,04$ & $0,12 \pm 0,03$ & $0,45 \pm 0,03$ & $0,42 \pm 0,03$ & $0,40 \pm 0,04$ & $0,33 \pm 0,05$ \\
$.50-.60$ & $0,23 \pm 0,05$ & $0,15 \pm 0,04$ & $0,55 \pm 0,03$ & $0,52 \pm 0,03$ & $0,49 \pm 0,04$ & $0,42 \pm 0,05$ \\
$.60-.70$ & $0,25 \pm 0,06$ & $0,19 \pm 0,03$ & $0,65 \pm 0,03$ & $0,62 \pm 0,03$ & $0,60 \pm 0,04$ & $0,51 \pm 0,05$ \\
$.70-.80$ & $0,36 \pm 0,10$ & $0,28 \pm 0,12$ & $0,77 \pm 0,03$ & $0,74 \pm 0,03$ & $0,70 \pm 0,04$ & $0,63 \pm 0,05$ \\
$.80-.90$ & $0,49 \pm 0,15$ & $0,42 \pm 0,17$ & $0,84 \pm 0,03$ & $0,83 \pm 0,03$ & $0,80 \pm 0,05$ & $0,75 \pm 0,07$ \\
$>.90$ & $0,43 \pm 0,12$ & $0,34 \pm 0,11$ & $0,92 \pm 0,00$ & $0,90 \pm 0,01$ & $0,88 \pm, 002$ & $0,84 \pm 0,03$ \\
Média & $0,21 \pm 0,16$ & $0,16 \pm 0,14$ & $0,49 \pm 0,23$ & $0,47 \pm 0,22$ & $0,46 \pm 0,22$ & $0,40 \pm 0,21$ \\
Mean & & & & &
\end{tabular}

${ }^{1}$ REL5 ${ }_{\text {animal }}=$ Confiabilidade estimada usando modelo animal e até cinco lactações por vaca (REL estimated using animal model and up to five lactation per cow).

REL5 ${ }_{m c r} \quad=$ REL estimada usando o método das companheiras de rebanho e até cinco lactações por vaca (REL estimated using a herdmate comparison method and up to five lactation per cow).

$\mathrm{REL5}_{\text {touro }}=\mathrm{REL}$ estimada usando modelo touro e até cinco lactações por vaca (REL estimated using sire model and up to five lactacions per cow).

$\mathrm{REL1}$ animal = REL estimada usando modelo animal e a produção de leite na primeira lactação (REL estimated using animal model and the milk production in the first lactation).

REL1 1 mcr = REL estimada usando MCR e a produção de leite na primeira lactação (REL estimated using the herdmate comparison method and the milk production in the first lactation).

$\mathrm{REL} 1_{\text {touro }}=\mathrm{REL}$ estimada usando modelo touro e a produção de leite na primeira lactação (REL estimated using sire model and the milk production in the first lactation).

ção na primeira lactação (PTA $1_{\text {touro }}$ e REL1 $\left.1_{\text {touro }}\right)$. Os valores são expressos por classe de confiabilidade estimada usando modelo animal, considerando até cinco lactações por vaca. Usando-se MA ou MT, verificou-se que as médias das PTA elevaram-se com o aumento da confiabilidade, o mesmo não foi observado para as PTA calculadas usando o MCR, cujas médias foram inconsistentes, segundo classes

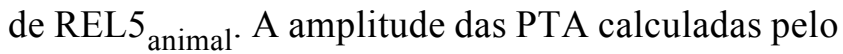
MCR foi menor que as obtidas quando se usaram modelos mistos, MA ou MT. Observa-se também que as médias de PTA para os 529 touros avaliados foram maiores e positivas para os modelos mistos, PTA5 animal, PTA $1_{\text {animal }}$ PTA5 $5_{\text {touro }}$ e PTA $1_{\text {touro }}$ e negativas para o MCR. Isto sugere que os previsores obtidos pelo MCR, se utilizados na seleção de touros, subestimariam os ganhos genéticos por geração. As confiabilidades médias estimadas, quando se usou MA ou MT, foram superiores às estimadas pelo MCR, indicando que MA e MT usam mais informações para avaliar o touro que o MCR.

Todavia, as confiabilidades das avaliações pelo modelo animal foram, para a maioria das classes, de duas a três vezes maiores que as obtidas usando-se as avaliações pelo MCR, foram apenas ligeiramente maiores em relação às obtidas pelo modelo touro. Os resultados apresentados na Tabela 3, por classe de confiabilidade das avaliações pelo MCR, com até cinco lactações por vaca, mostram que são poucos os touros (4\%) que têm confiabilidade maior que 0,6. Porém, quando se usou modelo animal, esta porcentagem foi superior a $46 \%$. Observa-se ainda que a confiabilidade máxima obtida pelo MCR foi de apenas 0,73 , enquanto para o MA e MT valores máximos de confiabilidades foram de 0,93 e 0,92 , respectivamente. Verifica-se que diferenças em médias de confiabilidades, entre MCR, MA e MT, é maior para as classes de menor valor de confiabilidade, em que há grande número de touros. Estes resultados concordam com os citados na literatura (NORMAN et al.,1991; VAN VLECK, 1992; e SCHAEFFER, 1996).

As correlações de ordem entre as PTA, previstas pelos diferentes métodos e modelos, incluindo até cinco ou apenas uma lactação por vaca oscilaram de 0,55 a 0,96 (Tabela 4). As correlações de Pearson foram similares e oscilaram de 0,57 a 0,96 . É interessante observar que, à medida que se reduz a quantidade de informações (menor número de lactações e de parentes), as correlações diminuem. Por exemplo, a correlação é de 0,96 entre as ordens e os valores obtidos no MA e MT com até cinco lactações por vaca; 0,79 entre as PTA calculadas usando MA considerando até cinco lactações ou a primeira lactação; e 0,60 entre PTA calculadas usando MA e o MCR com apenas a primeira lactação. Mesmo usando modelo animal, mantendo-se a mesma matriz de parentesco, observa-se que a quantidade de infor- 
VERNEQUE et al.

Tabela 3 - Médias de confiabilidade estimadas por diferentes procedimentos, por classe de confiabilidade calculada pelo método das companheiras de rebanho

Table 3 - Means for reliability estimated by different procedures, by classes of reliability calculated using contemporary herdmate comparison method

\begin{tabular}{|c|c|c|c|c|c|c|c|}
\hline \multirow[t]{2}{*}{$\begin{array}{l}\text { Classe de confiabilidade } \\
\text { Class of reliability }\end{array}$} & \multirow[t]{2}{*}{$\begin{array}{l}\mathrm{N}^{0} \text { de touros } \\
\text { No. of sires }\end{array}$} & \multicolumn{5}{|c|}{ Confiabilidade } & \multirow[b]{2}{*}{ REL1 $_{\text {touro }}$} \\
\hline & & $\mathrm{REL}_{\mathrm{mcr}}$ & $\mathrm{REL}_{\mathrm{mcr}}$ & REL5 $_{\text {animal }}$ & $\mathrm{REL}_{\text {animal }}$ & $\mathrm{REL5}_{\text {touro }}$ & \\
\hline$\leq .10$ & 150 & $0,07 \pm 0,01$ & $0,05 \pm 0,01$ & $0,26 \pm 0,05$ & $0,25 \pm 0,05$ & $0,25 \pm 0,05$ & $0,21 \pm 0,04$ \\
\hline $.11-.20$ & 138 & $0,15 \pm 0,03$ & $0,10 \pm 0,03$ & $0,40 \pm 0,10$ & $0,36 \pm 0,10$ & $0,38 \pm 0,10$ & $0,30 \pm 0,09$ \\
\hline $.21-.30$ & 133 & $0,25 \pm 0,03$ & $0,17 \pm 0,04$ & $0,62 \pm 0,14$ & $0,56 \pm 0,14$ & $0,60 \pm 0,14$ & $0,48 \pm 0,14$ \\
\hline $.31-.40$ & 46 & $0,35 \pm 0,03$ & $0,24 \pm 0,04$ & $0,74 \pm 0,12$ & $0,67 \pm 0,13$ & $0,72 \pm 0,12$ & $0,60 \pm 0,14$ \\
\hline $.41-.50$ & 17 & $0,46 \pm 0,03$ & $0,34 \pm 0,09$ & $0,81 \pm 0,07$ & $0,75 \pm 0,07$ & $0,79 \pm 0,07$ & $0,79 \pm 0,09$ \\
\hline $.51-.60$ & 24 & $0,56 \pm 0,03$ & $0,50 \pm 0,08$ & $0,83 \pm 0,04$ & $0,81 \pm 0,04$ & $0,82 \pm 0,04$ & $0,86 \pm 0,05$ \\
\hline $.61-.70$ & 18 & $0,64 \pm 0,03$ & $0,59 \pm 0,05$ & $0,84 \pm 0,03$ & $0,82 \pm 0,03$ & $0,82 \pm 0,03$ & $0,78 \pm 0,03$ \\
\hline $.71-.80$ & 4 & $0,72 \pm 0,03$ & $0,65 \pm 0,05$ & $0,90 \pm 0,02$ & $0,87 \pm 0,01$ & $0,89 \pm 0,02$ & $0,83 \pm 0,02$ \\
\hline Total & 529 & $0,21 \pm 0,16$ & $0,16 \pm 0,03$ & $0,49 \pm 0,23$ & $0,47 \pm 0,22$ & $0,46 \pm 0,22$ & $0,40 \pm 0,21$ \\
\hline Mínimo & - & 0,05 & 0,05 & 0,19 & 0,18 & 0,18 & 0,16 \\
\hline \multicolumn{8}{|l|}{ Minimum } \\
\hline Máximo & - & 0,73 & 0,71 & 0,93 & 0,92 & 0,92 & 0,88 \\
\hline Maximum & & & & & & & \\
\hline
\end{tabular}

Tabela 4 - Coeficientes de correlação de ordem (acima da diagonal) e correlação de Pearson (abaixo da diagonal) entre as PTA previstas pelos diferentes procedimentos

Table 4 - Rank correlation coefficients (upper diagonal) and correlation of Pearson (lower diagonal) between PTA, predicted by different procedures

\begin{tabular}{lcccccc}
\hline & $\mathrm{PTA5}_{\text {animal }}$ & $\mathrm{PTA}_{\text {mcr }}$ & $\mathrm{PTA}_{\text {touro }}$ & $\mathrm{PTA1}_{\text {animal }}$ & $\mathrm{PTA}_{\text {mcr }}$ & $\mathrm{PTA1}_{\text {touro }}$ \\
\hline PTA5 $_{\text {animal }}$ & - & 0,69 & 0,96 & 0,79 & 0,60 & 0,76 \\
PTA5 $_{\text {mcr }}$ & 0,68 & - & 0,73 & 0,55 & 0,74 & 0,59 \\
PTA $_{\text {touro }}$ & 0,96 & 0,72 & - & 0,76 & 0,62 & 0,79 \\
PTA1 $_{\text {animal }}$ & 0,84 & 0,57 & 0,80 & - & 0,68 & 0,94 \\
PTA $_{\text {mcr }}$ & 0,61 & 0,76 & 0,64 & 0,70 & - & 0,73 \\
PTA1 $_{\text {touro }}$ & 0,81 & 0,61 & 0,83 & 0,95 & 0,75 & - \\
\hline
\end{tabular}

mações influenciou a correlação, pois esta foi de 0,79 e 0,84 , respectivamente, para correlação de ordem e de valores entre PTA $5_{\text {animal }}$ e PTA $1_{\text {animal }}$. Na Tabela 5 são apresentadas as correlações de ordem da PTA, por classe de REL5 animal $_{\text {e as PTA obtidas }}$ pelos diferentes procedimentos. Tanto os resultados da Tabela 4 quanto os da Tabela 5 indicam que as classificações apresentaram-se altamente associadas quando se usou o modelo animal e modelo touro. Por outro lado, as correlações das PTA foram medianas ao se compararem os resultados obtidos usando-se MA e MCR, mas foram mais altas para baixos valores de confiabilidade (Tabela 5), indicando que, nesses casos, os modelos utilizaram igualmente as poucas informações disponíveis na avaliação dos touros, obtendo-se altos valores de correlação de ordem. Estas estimativas de correlação são similares às encontradas por VERNEQUE et al. (1996), mas são inferiores às citadas por NORMAN et al. (1991) e RUVUNA et al. (1973). Estas diferenças, possivelmente, estão relacionadas com o número de touros e de filhas por touro, por classe de confiabilidade que nos dados deste trabalho foi bem reduzido.

As diferenças absolutas, por classe de confiabilidade, entre os valores de PTA, previstos pelos diferentes procedimentos encontram-se na Tabela 6. Pode-se observar que essas diferenças crescem com o aumento da confiabilidade, confirmando os resultados obtidos pelas correlações entre os valores apresentados na Tabela 5. Em geral, as menores diferenças entre os valores foram para os modelos que utilizam da mesma quantidade de informação e têm a mesma base técnica. Assim, observase que a menor diferença absoluta total foi de $20,8 \mathrm{~kg}$ entre os valores previstos pelo modelo animal e modelo touro com até cinco lactações por vaca, seguido da diferença de 45,0 kg entre os modelos animal com até cinco lactações ou apenas uma lactação por vaca. A diferença máxima entre PTA5 $5_{\text {animal }}$ e PTA $5_{\text {mcr }}$ foi de $301,9 \mathrm{~kg}$, sendo que $15 \%$ dos touros apresentaram diferenças absolutas superiores a $100 \mathrm{~kg}$ (Tabela 7). Para PTA5 ${ }_{\text {animal }}$ e PTA ${ }_{\text {mcr }}$ a 
310 Rev. bras. zootec.

Tabela 5 - Coeficientes de correlação de ordem entre as PTA calculadas pelo modelo animal, por classe de confiabilidade, e as PTA previstas pelo método das companheiras de rebanho e pelo modelo touro

Table 5 - Rank correlation coefficient between PTA calculated by animal model, by classes of reliability, and PTA predicted by herdmate contemporary method and sire model

\begin{tabular}{|c|c|c|c|c|c|c|}
\hline \multirow[t]{2}{*}{$\begin{array}{l}\text { Classe de confiabilidade } \\
\text { Class of reliability }\end{array}$} & \multirow[t]{2}{*}{$\begin{array}{l}\mathrm{N}^{0} \text { de touros } \\
\text { No. of sires }\end{array}$} & \multicolumn{5}{|c|}{$\begin{array}{l}\text { Correlação de ordem } \\
\text { Rank correlation }\end{array}$} \\
\hline & & $\overline{\text { PTA5 }}_{\text {mcr }}$ & PTA1 ${ }_{\mathrm{mcr}}$ & PTA $1_{\text {animal }}$ & $\mathrm{PTA5}_{\text {touro }}$ & PTA $1_{\text {touro }}$ \\
\hline$<.20$ & 4 & 0.80 & 0,80 & 1,00 & 1,00 & 1,00 \\
\hline $.20-.30$ & 152 & 0.84 & 0,83 & 0,84 & 0,98 & 0,84 \\
\hline $.30-.40$ & 78 & 0.85 & 0,80 & 0,85 & 0,97 & 0,81 \\
\hline $.40-.50$ & 58 & 0.83 & 0,82 & 0,79 & 0,96 & 0,78 \\
\hline $.50-.60$ & 56 & 0.82 & 0,60 & 0,67 & 0,95 & 0,68 \\
\hline $.60-.70$ & 58 & 0,78 & 0,71 & 0,79 & 0,96 & 0,78 \\
\hline $.70-.80$ & 44 & 0,55 & 0,41 & 0,84 & 0,96 & 0,78 \\
\hline $.80-.90$ & 69 & 0,47 & 0,38 & 0,77 & 0,88 & 0,69 \\
\hline$>.90$ & 10 & 0,73 & 0,66 & 0,90 & 0,92 & 0,79 \\
\hline Total & 529 & 0,69 & 0,60 & 0,79 & 0,96 & 0,76 \\
\hline
\end{tabular}

Tabela 6 - Diferença absoluta média de PTA calculadas pelos diferentes procedimentos, por classes de confiabilidade estimada usando modelo animal

Table 6 - Mean of absolute difference in PTA calculated for different procedures, by classes of reliability estimated using animal model Classe de confiabilidade Média \pm Desvio-padrão

Classe of reliability Mean \pm Standard deviation

\begin{tabular}{|c|c|c|c|c|c|}
\hline & $\begin{array}{l}\mid \text { PTA5 animal } \\
\text { PTA } 5_{\text {mcr }} \mid\end{array}$ & 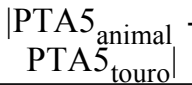 & $\begin{array}{l}\mid \text { PTA5 }{ }_{\text {animal }} \\
\text { PTA } 1_{\text {mer }} \mid\end{array}$ & $\begin{array}{l}\mid \text { PTA5 }_{\text {animal }}{ }^{-} \\
\text {PTA1 }_{\text {animal }}\end{array}$ & $\begin{array}{r}\left|\begin{array}{l}\text { PTA5 } \\
\text { PTAnimal } \\
\text { PTA } \\
\text { touro }\end{array}\right| \\
\end{array}$ \\
\hline$<.20$ & $36,2 \pm 25$ & $2,0 \pm 1$ & $35,3 \pm 24$ & $8,5 \pm 4$ & $5,2 \pm 4$ \\
\hline $.20-.30$ & $21,2 \pm 25$ & $5,7 \pm 7$ & $20,2 \pm 21$ & $17,9 \pm 22$ & $19,7 \pm 21$ \\
\hline $.30-.40$ & $33,9 \pm 29$ & $12,7 \pm 14$ & $39,3 \pm 31$ & $35,9 \pm 27$ & $40,8 \pm 32$ \\
\hline $.40-.50$ & $49,6 \pm 38$ & $18,7 \pm 20$ & $54,6 \pm 40$ & $46,0 \pm 43$ & $53,3 \pm 43$ \\
\hline $.50-.60$ & $53,9 \pm 42$ & $28,0 \pm 26$ & $70,2 \pm 49$ & $57,2 \pm 49$ & $60,1 \pm 55$ \\
\hline $.60-.70$ & $65,2 \pm 62$ & $24,9 \pm 26$ & $74,5 \pm 62$ & $61,8 \pm 42$ & $66,9 \pm 48$ \\
\hline $.70-.80$ & $103,6 \pm 86$ & $39,0 \pm 31$ & $116,0 \pm 95$ & $77,0 \pm 53$ & $83,3 \pm 63$ \\
\hline $.80-.90$ & $139,5 \pm 93$ & $42,3 \pm 30$ & $148,9 \pm 96$ & $67,6 \pm 44$ & $90,2 \pm 51$ \\
\hline$>.90$ & $95,5 \pm 86$ & $46,7 \pm 20$ & $111,4 \pm^{\prime} 99$ & $69,3 \pm 59$ & $96,3 \pm 58$ \\
\hline Total & $57,9 \pm 66$ & $20,8 \pm 25$ & $64,5 \pm 71$ & $45,0 \pm 43$ & $51,5 \pm 49$ \\
\hline
\end{tabular}

Tabela 7 - Número e porcentagem de touros, por classes de diferença absoluta de PTA

Table 7 - Number and percentage of sires, by classes of absolute difference in PTA

\begin{tabular}{|c|c|c|c|c|c|c|c|c|c|c|}
\hline $\mathrm{DIF}^{\mathrm{a}}$ & & nimal & & ${ }_{\text {imal }}$ & $\begin{array}{l}\mid \mathrm{PT} \\
\mathrm{PT}\end{array}$ & al - & $\begin{array}{l}\mid \text { PTA } \\
\text { PTA } 1\end{array}$ & & & nimal \\
\hline$(\mathrm{kg})$ & $\mathrm{N}$ & $\%$ & $\mathrm{~N}$ & $\%$ & $\mathrm{~N}$ & $\%$ & $\mathrm{~N}$ & $\%$ & $\mathrm{~N}$ & $\%$ \\
\hline Até $10 \mathrm{~kg}$ & 101 & 19,1 & 252 & 47,7 & 98 & 18,6 & 117 & 22,1 & 110 & 20,8 \\
\hline $11-20$ & 94 & 17,8 & 86 & 16,3 & 80 & 15,2 & 87 & 16,5 & 66 & 12,4 \\
\hline $21-30$ & 59 & 11,1 & 62 & 11,7 & 67 & 12,6 & 67 & 12,7 & 57 & 10,8 \\
\hline $31-40$ & 37 & 7,0 & 33 & 6,3 & 45 & 8,5 & 46 & 8,7 & 58 & 10,9 \\
\hline $41-50$ & 48 & 9,0 & 26 & 4,8 & 30 & 5,7 & 30 & 5,7 & 30 & 5,7 \\
\hline $51-100$ & 91 & 17,2 & 61 & 11,5 & 87 & 16,5 & 115 & 21,7 & 119 & 22,5 \\
\hline $101-150$ & 39 & 7,4 & 9 & 1,7 & 57 & 10,7 & 67 & 12,6 & 89 & 16,9 \\
\hline $151-250$ & 30 & 5,7 & & & 28 & 5,3 & & & & \\
\hline$>250$ & 30 & 5,7 & & & 35 & 6,7 & & & & \\
\hline Total & & & & & & & & & & \\
\hline Dif.mínima & & & & & & & & & & \\
\hline Minimum dif. & & & & & & & & & & \\
\hline Dif.máxima & & & & & & & & & & \\
\hline Maximum dif. & & & & & & & & & & \\
\hline
\end{tabular}




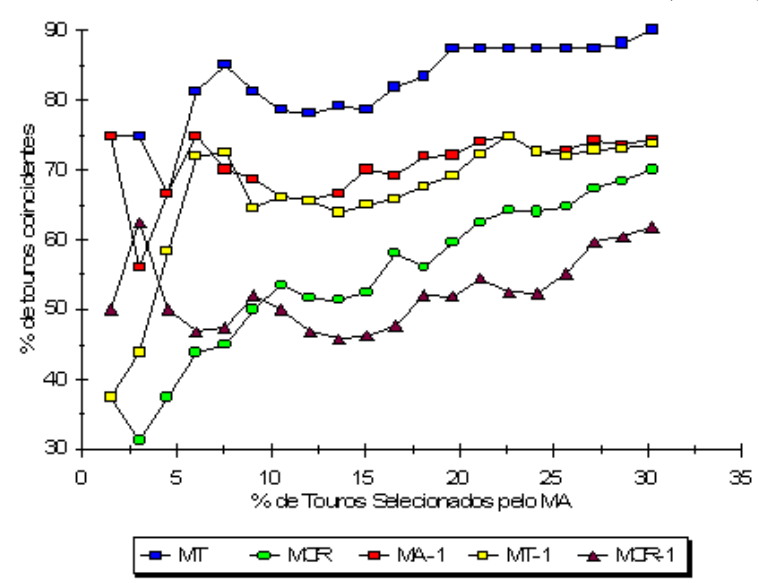

Situação 1 - Confiabilidade $=0,49$ Situation 1 - Reliability $=0,49$

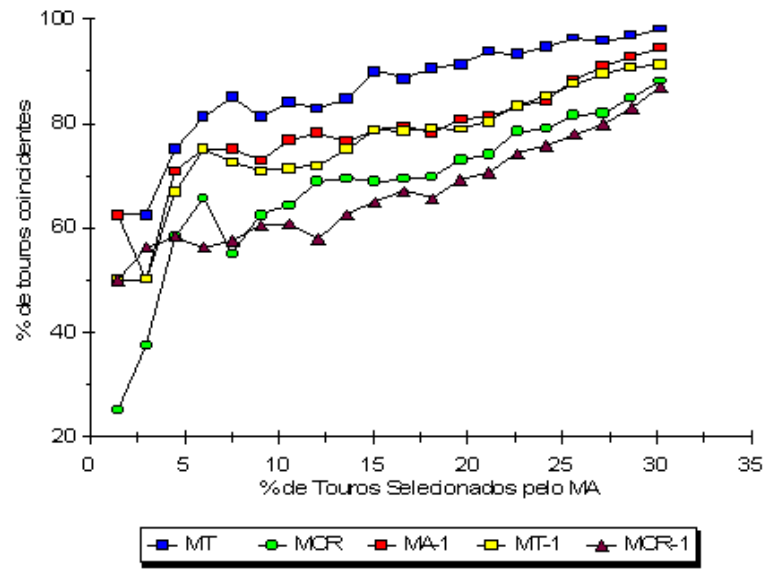

Situação 2 - Confiabilidade $>0,60$ Situation 2 - Reliability $>0,60$

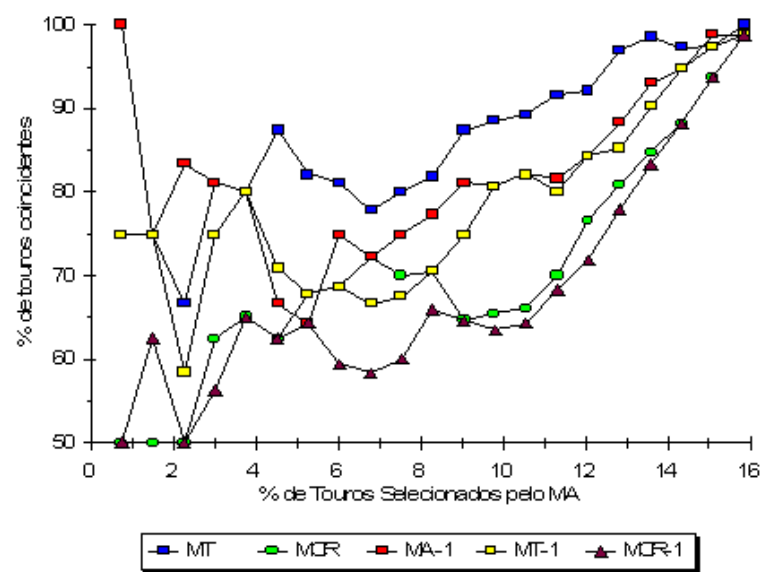

Situação 3 - Confiabilidade $>0,80$

Situation 3 - Reliability $>0,80$

Figura 1 - Porcentagem de coincidência (escolha dos mesmos touros pelo modelo animal versus pelos demais modelos). Situação 1. Seleção de $30 \%$ dos melhores touros pelo MA, independente da confiabilidade de prova; Situação 2. Seleção de até $30 \%$ dos melhores touros pelo MA com confiabilidade superior a $60 \%$; Situação 3 . Seleção dos melhores touros pelo MA dentre aqueles com confiabilidade superior a $80 \%$.

Figure 1 - Percentage of coincidence (choice of the best sires by animal model versus other models). Situation 1 . Selection of $30 \%$ of the best sires by MA, independent of reliability of the test; Situation 2. Selection up to $30 \%$ of the best sires by animal model with reliability greater than 60\%; Situation 3. Selection up to 30\% of the best sires using animal model with reliability greater than $80 \%$.

diferença máxima foi de $362,6 \mathrm{~kg}$, equivalente a $19 \%$ dos touros (Tabela 7). Por outro lado, comparando-se as PTA obtidas pelo modelo animal com as do modelo touro, observou-se que apenas $1,1 \%$ dos touros apresentam diferença absoluta superior a $100 \mathrm{~kg}$ e $49,2 \%$ apresentaram diferença em PTA inferior a $10 \mathrm{~kg}$. Estes resultados indicam alto grau de similaridade entre avaliações usando-se o MA ou MT, o que é esperado, pois em características com herdabilidade próxima a 0,25 o peso das informações da progênie na avaliação do touro, usando-se MA ou MT, é aproximadamente $82 \%$ (SCHAEFFER, 1996). No presente trabalho, na avaliação de touro usando-se o MA ou MT, além de produções das filhas, foram agregadas outras informações de pedigree.

Na Figura 1 são apresentados os gráficos indicando o percentual de coincidência de touros selecionados quando foram usados o modelo touro, o método das companheiras de rebanho e modelo animal, com a produção na primeira lactação, considerandose a classificação básica de touro pela PTA calculada 
pelo modelo animal, usando-se até cinco lactações por vaca. A primeira situação refere-se à condição em que se selecionaram até $30 \%$ dos touros de uma amostra de 529 animais (REL5 ${ }_{\text {animal }}$ média de 0,49). Na situação 2 foram selecionados até $30 \%$ dos touros melhores classificados usando-se MA entre os touros com REL5 animal superior a 0,60. Na terceira situação consideraram-se apenas 79 touros (15\%) classificados com REL5 animal superior a 0,80 . Observa-se que, usando-se a PTA5animal, o percentual de coincidência com touros classificados pelo MA e MCR ou MA e MCR considerando-se apenas a produção no primeiro parto foi inferior aos obtidos, na mesma situação, pelo MA ou MT. Selecionando-se, por exemplo, $20 \%$ dos melhores touros com confiabilidade maior que 0,60 avaliados pelo MA, 90 e $65 \%$ destes seriam também selecionados se fossem usados o MT ou $\mathrm{MCR}$, respectivamente. Isto significa que, considerando-se o modelo animal como o adequado, porque os previsores de valores genéticos apresentam maior média de confiabilidade, a margem de erro na avaliação de touros usando-se MCR foi alta. Esta foi ainda maior quando a avaliação foi realizada considerandose apenas a produção de leite na primeira lactação, pois o MCR usa menor número de informações para avaliar touro e vaca.

\section{Conclusões}

Usando-se metodologia adequada, pode-se conseguir maiores ganhos genéticos por geração, pela melhor seleção de touros segundo a confiabilidade e maior média de PTA.

As capacidades previstas de transmissão para touros, avaliados por meio do modelo animal ou modelo touro, com informação de parentes, sempre apresentaram maior confiabilidade que a obtida pelo método das companheiras de rebanho.

A maior eficiência dos modelos mistos na obtenção de PTA, usando-se o modelo animal ou modelo touro, sugere que esses devem ser ratificados como os melhores modelos a serem considerados nos programas de avaliação genética.

\section{Agradecimentos}

Aos criadores colaboradores do programa de teste de progênie. Aos idealizadores do programa de melhoramento genético do Gir leiteiro.

\section{Referências Bibliográficas}

BOLDMAN, K.G., VAN VLECK, L.D. 1991. Derivative-free restricted maximum likelihood estimation in animal models with a sparse matriz solver. J. Dairy Sci., 74(12):4337-4343.

BOLDMAN, K.G., KRIESE, L.A., VAN VLECK, L.D. et al. 1995. A manual for use of MTDFREML: a set of programs to obtain estimates of variances and covariances [DRAFT]. Lincoln: Department of Agriculture/ Agriculture Research Service, $120 \mathrm{p}$.

CONOVER, W.J. 1980. Pratical nonparametric statistics. 2. ed. New York: John Wiley, 493p.

DOMMERHOLT J. 1982. Sire and cow evaluation program. Coronel Pacheco: Embrapa- CNPGL, 42p. ( mimeo)

HENDERSON, C.R. Sire evaluation and genetic trends. In: ANIMAL BREEDING AND GENETICS SYMPOSIUM, 10., 1973, Champaign. Proceedings... Menasha: American Society of Animal Science, p. 41, 1973.

HENDERSON, C.R. 1989. Theoretical bases and computational methods for a number of differents animal models. J. Dairy Sci., 71:1-16, suppl. 2.

MEYER, K. 1988. A set of programs to estimate variance components under individual animal model. J. Dairy Sci., 71(2):33-34.

NORMAN, H. D., WIGGANS, G.R., ERNST, C.A. et al. 1989. Determination of size of management for optimizing predictability in the animal model. J. Dairy Sci., 72(1):71, suppl 1.

NORMAN, H.D., POWELL, R.L., WIGGANS, G.R. 1991. Comparison of genetic evaluations from animal model and modified contemporary comparison. J. Dairy Sci., 74(7):2309-2316.

RUVUNA, F., MAO, I.L., MEADOWS, C.E. 1973. A semisimulation approach. I. Comparison of sire evaluation methods. In: ANNUAL MEETING OF AMERICAN DAIRY SCIENCE ASSOCIATION, 74, 1979, Logan: American Dairy Science Association. Anais... Logan, p. 1-6.

SCHAEFFER, L. Animal models: why, how and when to uso them. In: SIMPÓSIO NACIONAL DE MELHORAMENTO ANIMAL, 1, 1996, Ribeirão Preto. Anais...Ribeirão Preto, p. 21-40, 1996.

VAN VLECK, L.D. Animal model for bull and cow evaluation. In: LARGE DAIRY HEARD MANAGEMENT SYMPOSIUM, 1992, Nebrasca. Proceedings...Nebrasca, p. 1-31, 1992.

VANRADEN, P.M., ERNST, C. A., WIGGANS, G.R. et al. 1989. Ability of animal model and modifield conteporary comparison evaluations of parents to predict future progeny performance. J. Dairy Sci., 72(1):72.

VERNEQUE, R.S. Procedimentos numéricos e estimação de componentes de covariância em análise multivariada pelo método da máxima verossimilhança restrita: modelos mistos aplicados ao melhoramento animal. Piracicaba, SP, ESALQ, 1994. 155p. Tese (Doutorado em Bioestatística) - Escola Superior de Agricultura Luiz de Queiroz, 1994.

VERNEQUE, R. S., PIMENTEL, A. A., FERREIRA, W. J. et al. Avaliação genética de vacas e touros pelo modelo animal e pelo método das companheiras de rebanho em animais da raça "Gir Leiteiro". In: REUNIÃO ANUAL DA SOCIEDADE BRASILEIRA DE ZOOTECNIA, 33, 1996, Fortaleza. Anais... Fortaleza: SBZ, p. 27-29, 1996.

Recebido em: 04/12/97 Aceito em: 18/08/98 